\title{
Validade Fatorial do Questionário de Saúde Geral (QSG-28)
}

\author{
Camila Teixeira Heleno ${ }^{1}$ (D) \\ Universidade Federal dos Vales do Jequitinhonha e Mucuri, Diamantina-MG, Brasil \\ Livia de Oliveira Borges (D) \\ Universidade Federal de Minas Gerais, Belo Horizonte-MG, Brasil \\ Esteban Agulló-Tomás (iD \\ Universidad de Oviedo, Oviedo/AS, Espanha
}

\section{RESUMO}

Conduzimos pesquisa com objetivo de avaliar a validade fatorial e a consistência interna do Questionário de Saúde Geral de Goldberg com 28 itens (QSG-28) no Brasil, comparando os ajustes de sua estrutura fatorial com quatro, três e cinco fatores. Realizamos procedimento de tradução reversa (inglês-português-inglês) do instrumento. Aplicamos o QSG-28 em 878 estudantes de duas faculdades de Belo Horizonte/MG. Desenvolvemos análise fatorial exploratória, Smallest Space Analysis e análise fatorial confirmatória (AFC). Na AFC, nenhum dos modelos apresentou bons ajustes. O modelo de três fatores do QSG-28 se mostrou mais adequado que os modelos de quatro e cinco fatores para a avaliação de estudantes universitários, ainda que o modelo de quatro fatores possa ser adotado. Concluímos que o instrumento se configura em uma alternativa para rastrear alterações na saúde mental em indivíduos não clínicos, podendo ser utilizado em estudos no campo do trabalho.

Palavras-chave: QSG-28; análise fatorial confirmatória; estudantes universitários.

ABSTRACT - Factorial validity of the Goldberg General Health Questionnaire (GHQ-28)

This study evaluated the factorial validity and internal consistence of the Brazilian version of the 28-item Goldberg General Health Questionnaire (QSG-28), comparing the four, three and five factor adjustments of its factorial structure. A reverse translation procedure (English-Portuguese-English) of the instrument was carried out. We applied the QSG-28 with 878 students from two colleges in Belo Horizonte/MG. Exploratory factorial analysis, Smallest Space Analysis and confirmatory factorial analysis (CFA) were performed. In the CFA, none of the models presented a good fit. The three-factor model of the QSG-28 was more appropriate than the four- and five-factor models for the evaluation of college students, although the four-factor model could be adopted. It was concluded that the instrument is an alternative for screening for changes in mental health in non-clinical individuals and can be used in occupational studies.

Keywords: GHQ-28; confirmatory factor analysis; college students.

\section{RESUMEN - Validez factorial del Cuestionario de Salud General (GHQ-28)}

Se llevó a cabo una investigación con el objetivo de evaluar la validez factorial y consistencia interna del Cuestionario de Salud General de Goldberg con 28 ítems (QSG-28) en Brasil, comparando los ajustes de su estructura factorial con cuatro, tres y cinco factores. El procedimiento de traducción inversa (Inglés-Portugués-Inglés) del instrumento fue realizado. El QSG-28 fue aplicado en 878 estudiantes de dos facultades de Belo Horizonte/MG. También se realizó un análisis factorial exploratorio, Smallest Space Analysis y análisis factorial confirmatorio (AFC). En el AFC, ninguno de los modelos presentó buenos ajustes. El modelo de tres factores del QSG-28 se mostró más adecuado que los modelos de cuatro y cinco factores para la evaluación de estudiantes universitarios, aunque el modelo de cuatro factores pueda ser utilizado en estudios en el campo del trabajo. Concluimos que el instrumento se configura en una alternativa para el seguimiento de alteraciones en la salud mental en individuos no clínicos, pudiendo ser utilizado en estudios en el ámbito del trabajo.

Palabras clave: GHQ-28; análisis factorial confirmatorio; estudiantes universitarios.

As intervenções clínicas, especialmente as focalizadas na prevenção, incluindo aquelas na área da saúde do trabalhador, bem como as pesquisas epidemiológicas e de campo demandam o uso de instrumento de triagem e rastreamento, os quais devem abranger um número pequeno de itens e serem de aplicação simples (Wang, 
2016). Tal demanda foi o estímulo inicial para focalizar nosso interesse de pesquisa no Questionário de Saúde Geral (QSG), na sua versão de 28 itens. Ele tem sido utilizado em diferentes culturas/países com o intuito de rastrear alterações na saúde mental em populações não clínicas, incluindo estudos ocupacionais (p. ex., Kock, Görgens-Ekermans, \& Dhladhla, 2014; Makowska, Merecz, Moscicka, \& Kolasa, 2002; Suda, Nakayama, \& Morimoto, 2007).

\section{O QSG-28 e as Pesquisas Antecedentes}

O QSG ou General Health Questionnaire (GHQ) foi apresentado originalmente em 1972, por Goldberg, em sua versão mais ampla com 60 itens. O QSG foi concebido para identificar a incapacidade do indivíduo de realizar atividades usuais entre as pessoas e o aparecimento de fenômenos estressantes novos (Goldberg \& Hillier, 1979).

Existem várias versões do QSG com quantidades diferentes de itens (entre 12 e 60 itens), criadas a partir da versão ampla de 60 itens. A versão de 28 itens, QSG28, é também conhecida como Scaled GHQ (Goldberg \& Hillier, 1979), sendo considerada a única versão que fornece medidas de subescalas de domínios mais específicos da psicopatologia (Gibbons, Arévolo, \& Mónico 2004). Seu uso é indicado quando o investigador necessita de mais informação do que um simples escore de severidade (Goldberg \& Hillier, 1979). Tem como vantagens a rapidez e facilidade de aplicação e como desvantagens o fato de algumas escalas, como de sintomas psicossomáticos, não diferenciarem adequadamente entre os problemas físicos e os problemas psicológicos e não detectarem bem pacientes crônicos (Vallejo, Rivera, EsteveVives, Rodríguez-Muñoz, \& ICAF, 2014). Para construção dessa versão, a partir do QSG-60 foram realizadas análises estatísticas (como a análise fatorial pela técnica dos componentes principais com rotação varimax) que indicaram uma solução de quatro fatores, tendo sido selecionados sete itens que mais pontuavam em cada um. Trata-se de um instrumento cuja validade foi examinada de forma mista, ou seja, em sua versão original, foram considerados critérios clínicos e também psicométricos (Ribeiro et al., 2015).

Os quatro fatores do QSG-28 foram nomeados como: Sintomas Somáticos (SS), Ansiedade e Insônia (AI), Disfunção Social (DS) e Depressão Grave (DG). Esses fatores se mostraram correlacionados entre si (Gibbons et al., 2004; Goldberg \& Hillier, 1979; Werneke, Goldberg, Yalcin, \& Üstün, 2000), correspondendo, desse modo, a indícios sintomatológicos e não necessariamente diagnósticos distintos. Gibbons, Arévolo e Mónico (2004), Kock, Görgens-Ekermans e Dhladhla (2014) e Werneke, Goldberg, Yalcin e Üstün (2000), por exemplo, sustentaram que a proximidade do fator Sintomas Somáticos ao de Ansiedade e Insônia sugeriria uma estrutura de três fatores, ainda que a estrutura de quatro fatores seja mais encontrada na literatura (p. ex., Javanmard \& Mamaghani, 2013; Malakouti, Fatollahi, Mirabsadeh, \& Zandi, 2006; Vallejo et al., 2014).

A primeira versão do QSG-28 explicou 59\% da variância total (Goldberg \& Hillier, 1979), com variações em aplicações posteriores em diferentes países apresentadas na literatura, como 54\% em El Salvador (Gibbons et al., 2004); 60,24\% em Portugal (Ribeiro et al., 2015); 49,37\% no Irã (Javanmard \& Mamaghani, 2013); 55,4\% na Espanha (Delgado-Gomez et al., 2013) e 61\% em 15 cidades do mundo (Werneke et al., 2000). De modo geral, os resultados têm sido satisfatórios para aplicação com diferentes populações (clínicas e não clínicas) em termos de consistência interna do instrumento, como: El Salvador $\left(\alpha_{\mathrm{SS}}=0,81, \alpha_{\mathrm{AI}}=0,86, \alpha_{\mathrm{DS}}=0,79, \alpha_{\mathrm{DG}}=0,87\right.$ e $\left.\alpha_{\text {total }}=0,86\right)$ (Gibbons et al., 2004), Portugal $\left(\alpha_{\mathrm{SS}}=0,89\right.$, $\alpha_{\mathrm{AI}}=0,89, \alpha_{\mathrm{DS}}=0,86, \alpha_{\mathrm{DG}}=0,89$ e $\alpha_{\text {total }}=0,94$ ) (Ribeiro et al., 2015), Irã ( $\alpha_{\mathrm{SS}}=0,78, \alpha_{\mathrm{Al}}=0,61, \alpha_{\mathrm{DS}}=0,60, \alpha_{\mathrm{DG}}=0,84$ e $\left.\alpha_{\text {total }}=0,87\right)$ (Javanmard \& Mamaghani, 2013), Espanha $\left(\alpha_{S S}=0,79, \alpha_{A I}=0,90, \alpha_{D S}=0,79, \alpha_{D G}=0,91\right.$ e $\alpha_{\text {total }}=0,94$; $\alpha_{\mathrm{SS}}=0,83, \alpha_{\mathrm{AI}}=0,86, \alpha_{\mathrm{DS}}=0,86, \alpha_{\mathrm{DG}}=0,83$ e $\left.\alpha_{\mathrm{total}}=0,94\right)$ (Vallejo et al., 2014; Pérez, Lozano, \& Rojas, 2010, respectivamente), Líbano ( $\alpha$ entre 0,90 e 0,91 ) (Farhood \& Dimassi, 2015) e na África do Sul ( $\alpha$ total $=0,70$ a 0,83, analisando modelos de três, quatro e cinco fatores) (Kock et al., 2014).

Em relação ao uso do QSG-28 no Brasil, foi localizada uma pesquisa realizada no Rio de Janeiro (dados coletados em 1991 e 1992) com 2.795 pacientes de um hospital universitário (Villano, Nanhay, Moraes, \& Costa e Silva, 1995). Essa pesquisa fez parte de um estudo da Organização Mundial de Saúde (OMS) realizado em 15 cidades pelo mundo (de 14 países e em 11 línguas diferentes), com cerca de 30 mil participantes, tendo o objetivo de levantar a frequência de transtornos mentais nos serviços de atenção primária em saúde, em diferentes países, desenvolvidos e em desenvolvimento (Üstün \& Sartorius, 1995). Algumas publicações derivadas desse estudo (p. ex., Goldberg et al., 1997; Werneke et al., 2000), que incluíram os dados cariocas, objetivaram comparar o QSG-28 e o QSG-12 e analisar a estabilidade da estrutura fatorial de ambos instrumentos. Werneke et al. (2000) indicaram uma solução de cinco fatores para o Rio de Janeiro, sem especificar quais os ajustes obtidos e quais itens comporiam cada fator, inviabilizando tomá-la como ponto de partida para a realização de uma análise fatorial confirmatória (AFC). Ainda assim, os autores optaram por analisar o instrumento com quatro fatores, conforme versão original, permitindo a comparação com as demais cidades investigadas (como Santiago e Berlim). Considerando os quatro fatores, o estudo na cidade teve uma variância explicada de 60,3\% (a variação entre as 15 cidades foi de 50,6\% em Ibadan, na Nigéria, e 73,2\% em Bangalore, na Índia), utilizando uma análise fatorial com rotação varimax. Adicionalmente, os autores relataram que a solução de quatro fatores explicou $11 \%$ 
menos, comparando as amostras das décadas de 1970 e 1990, sinalizando transformações na estrutura fatorial do QSG-28.

AFCs do QSG-28 se tornaram mais comuns a partir da década de 2010 (p. ex., Ardakani et al., 2016; Kock et al., 2014; Munyombwe, West, \& Hill, 2015; Pérez et al., 2010). Desses estudos, o de Kock et al. (2014), realizado na África do Sul, aplicou AFCs com três modelos distintos: com três, quatro e cinco fatores, indicando ajustes ligeiramente melhores para o primeiro modelo. O de Perez, Lozano e Rojas (2010), na Espanha, realizou análises com o modelo de quatro fatores e o de três fatores, encontrando ajustes melhores para o primeiro. Os outros dois estudos citados - Ardakani et al. (2016) e Munyombwe, West e Hill (2015) - na Malásia e no Reino Unido, realizaram AFCs apenas com o modelo de quatro fatores, tendo encontrado resultados que suportam essa estrutura (Tabela 1). Entretanto, em todas as análises confirmatórias, o ajuste do modelo é apenas moderado, apresentando indicadores favoráveis, mas com nível de significância do quiquadrado $(p<0,05)$ que predominantemente rejeita o bom ajuste.

Tabela 1

Índices de Ajustes de Análises Fatoriais Confirmatórias de Modelos Fatoriais do QSG-28

\begin{tabular}{|c|c|}
\hline Autores/ $\mathrm{N}^{\circ}$ de fatores & Índices de ajustes \\
\hline $\begin{array}{l}\text { Ardakani et al., } 2016 \\
\quad 4 \text { fatores }\end{array}$ & $\begin{array}{c}\chi^{2}=810,8 ; d f=305 ; p<0,001 ; \text { RMSEA }=0,05 \\
\text { CFI=0,962; GFI=0,918; AGFI=0,898; } \\
\text { Composite Reliability: } 0,915 \text { a 0,859 }\end{array}$ \\
\hline $\begin{array}{l}\text { Kock et al., } 2014 \\
\quad 4 \text { fatores }\end{array}$ & $\begin{aligned} \chi^{2}= & 85,66 ; d f=344 ; p<0,001 ; \operatorname{RMSEA}=0,036 \\
& \text { CFI=0,99; NNFI=0,99; SRMR=0,071 }\end{aligned}$ \\
\hline 5 fatores & $\begin{array}{l}\chi^{2}=854,15 ; d f=340 ; p<0,001 ; \text { RMSEA }=0,034 \\
\quad \text { CFI }=0,99 ; \text { NNFI=0,99; SRMR }=0,070\end{array}$ \\
\hline 3 fatores & $\begin{array}{c}\chi^{2}=888,67 ; d f=347 ; p<0,001 ; \text { RMSEA }=0,035 \\
\quad \text { CFI }=0,99 ; \text { NNFI }=0,99 ; \text { SRMR=0,072 }\end{array}$ \\
\hline $\begin{array}{l}\text { Munyombwe et al., } 2015 \\
\quad 4 \text { fatores }\end{array}$ & $\begin{array}{l}\text { Amostra 1: } \chi^{2}=740,79 ; p<0,001 ; \text { RMSEA }=0,048 ; \text { CFI }=0,958 ; \text { TLI }=0,954 \\
\text { Amostra 2: } \chi^{2}=490,71 ; p<0,001 ; \text { RMSEA }=0,035 ; \text { CFI }=0,969 ; \text { TLI }=0,965\end{array}$ \\
\hline $\begin{array}{l}\text { Perez et al., } 2010 \\
\quad 4 \text { fatores }\end{array}$ & $\begin{array}{c}\mathrm{SB} \chi^{2}=504,07 ; d f=339 ; p<0,001 ; \mathrm{RMSEA}=0,060 \\
\mathrm{CFI}=0,921 ; \mathrm{IFI}=0,923 ; \mathrm{GFI}=0,771 ; \mathrm{NFI}=0,797 ; \mathrm{NNFI}=0,912\end{array}$ \\
\hline 3 fatores & $\begin{array}{c}\mathrm{SB} \chi^{2}=524,55 ; d f=342 ; p<0,001 ; \mathrm{RMSEA}=0,062 ; \\
\mathrm{CFI}=0,913 ; \mathrm{IFI}=0,915 ; \mathrm{GFI}=0,764 ; \mathrm{NFI}=0,788 ; \mathrm{NNFI}=0,904\end{array}$ \\
\hline
\end{tabular}

Conforme o que já foi proposto anteriormente, é pertinente considerar, em síntese, que faltam pesquisas sobre o QSG-28 no país, para que seja utilizado com instrumento de triagem e rastreamento, e há divergências técnicas acerca de qual seja a estrutura fatorial mais adequada. Planejou-se, então, a presente pesquisa com o objetivo de avaliar a estrutura fatorial do QSG-28 no Brasil com três, quatro e cinco fatores, bem como levantar os coeficientes de consistência de cada fator, contribuindo para a obtenção de evidências de validade acerca da aplicação do instrumento.

\section{Método}

\section{Participantes}

Participaram da pesquisa 878 estudantes de diferentes cursos superiores (bacharelados e tecnólogos) das grandes áreas de Engenharias, Ciências Sociais Aplicadas e Ciências Humanas de duas faculdades privadas de
Belo Horizonte/MG. Do total, considerou-se 809 participantes que não apresentaram nenhuma resposta em branco para o QSG-28. Ainda rastreando a qualidade dos dados também se tomou a decisão de considerar válidos os questionários em que os participantes responderam no máximo a 23 itens com alternativas correspondentes a um mesmo escore. Levou-se em conta que autores (p. ex., Cea D'ancona, 2004; Gray, 2012; Tabachnick \& Fidell, 1989) têm assinalado a importância da qualidade dos bancos de dados. Enfim, a amostra totalizou 793 casos a serem analisados.

Os participantes apresentaram idade média de 28,15 anos $(D P=9,59), 56,4 \%(n=434)$ são do sexo feminino, têm renda média por pessoa de $\mathrm{R} \$ 1.320,38$ $(D P=1.527,37), 74,5 \%(n=591)$ são empregados.

\section{Instrumentos}

Ficha sociodemográfica e ocupacional. Abrangeu informações para descrever os participantes. 
QSG-28 (Goldberg \& Hillier, 1979). A versão adotada do QSG possui 28 questões prevendo quatro fatores com sete itens cada: Sintomas Somáticos (itens A1 a A7), Ansiedade e Insônia (itens B1 a B7), Disfunção Social (itens C1 a C7), e Depressão Grave (itens D1 a D7). Foi adotado o método de pontuação de respostas da escala Likert (0 a 3) (Goldberg et al., 1997). Desse modo, os escores são somados para fornecer a pontuação de cada fator (que varia de 0 a 21) e também a pontuação total (0 a 84). Quanto maior a pontuação pior a saúde mental do indivíduo.

\section{Procedimentos de Tradução do QSG-28}

Foi realizado um procedimento de tradução reversa (inglês-português-inglês) do QSG-28 a partir da versão original deste questionário (Goldberg \& Hillier, 1979). A tradução para o português foi realizada pelas pesquisadoras brasileiras e a tradução para o inglês por um pesquisador estrangeiro que domina as duas línguas. Comparouse a retrotradução com a versão original e analisamos/ solucionamos divergências. Também consideramos traduções do QSG realizadas anteriormente e já utilizadas no Brasil, a do QSG-60 (Pasquali, Gouveia, Andriola, Miranda, \& Ramos, 1994; Gouveia, Miranda, Ramos, Andriola, \& Pasquali, 1996) e do QSG-12 (Borges \& Argolo, 2002; Gouveia et al., 2003), por possuírem questões que se repetem nestas.

\section{Procedimento de Coleta de Dados}

A aplicação dos instrumentos foi realizada de forma coletiva por uma equipe previamente treinada pelas pesquisadoras, composta por estudantes de graduação e pós-graduação em Psicologia. Com anuência institucional e apoio dos professores, membros da equipe convidaram estudantes das faculdades privadas mencionadas a participar voluntariamente da pesquisa (critério de adesão), assinando um Termo de Consentimento Livre e Esclarecido (TCLE) destacado dos instrumentos de pesquisa e tendo o anonimato e confidencialidades preservadas pela sua não identificação. Esse projeto de pesquisa foi avaliado pelo Comitê de Ética em Pesquisa vinculado à Universidade Federal de Minas Gerais, sob o parecer $n^{\circ}$ CAAE 23470619.0.0000.5149.

\section{Procedimentos de Análise dos Dados}

Foram registadas as respostas dos participantes em banco de dados no Statistical Package for the Social Sciences (SPSS), para realização das análises estatísticas referentes à descrição da amostra, às análises preliminares dos dados, a análise fatorial exploratória (AFE) e a aplicação da Smallest Space Analysis (SSA). Optamos por iniciar realizando uma AFE porque um estudo (Werneke et al., 2000) realizado no Rio de Janeiro na década de 1990 sugeria uma estrutura de cinco fatores, sem especificar detalhadamente a composição destes. Para tanto, dos 793 participantes selecionamos aleatoriamente 280 deles para a etapa exploratória. Realizamos AFE em etapas: nas preliminares, avaliamos a normalidade das distribuições de respostas aos itens do QSG-28, a fatorabilidade e a adequação da amostra; na etapa final, aplicamos a AFE pela técnica dos eixos principais, com rotação oblíqua (por causa da conhecida correlação entre os fatores), especificando uma solução de cinco fatores.

Posteriormente, exportamos o banco de dados para o programa Lisrel (Linear Structural Relationships) para realização das AFCs (Jöreskog \& Sörbom, 1993; Malhorta, Lopes, \& Veiga, 2014). Consideramos as estruturas fatoriais já encontradas na literatura com quatro e três fatores e incluímos a estrutura de cinco fatores (tendo a AFE referida anteriormente como ponto de partida). Em síntese: o modelo de quatro fatores é a estrutura clássica do QSG-28 (Goldberg \& Hillier, 1979); o modelo de três fatores reúne os itens de Sintomas Somáticos e Ansiedade e Insônia em um único fator, nomeado como Estresse Psicológico (Kock et al., 2014); o modelo de cinco fatores inclui a divisão do fator Ansiedade e Insônia em dois, a inserção dos itens A3 e A2 no fator Ansiedade e supressão dos itens A7, C1, C2, D5. Essas diferenças de inclusão e exclusão de itens no modelo com cinco fatores ocorreu considerando os resultados da AFE conforme descreveremos na seção subsequente. Calculamos, também, os alfas de Cronbach para cada fator dos modelos.

Aplicamos, por fim, a SSA que é usual quando temos itens muito correlacionados entre si, de modo que passamos a contar com um terceiro caminho de análises para a consecução do objetivo proposto. Consideramos as publicações anteriores (Gibbons et al., 2004; Kock et al., 2014; Werneke et al., 2000) que indicaram correlações entre os fatores.

\section{Resultados}

Acerca da distribuição das respostas, analisamos os coeficientes Kolmogorov-Smirnov e houve rejeição da normalidade em todos os itens (Cea D'Ancona, 2004). Entretanto, levantamos também para cada item do QSG28 os coeficientes de assimetria e curtose, bem como histogramas. Apenas três itens (D3, D4, D7) apresentaram assimetria e/ou curtose maiores ou iguais a 2,0 (Damásio, 2012; Marôco, 2014). A ausência de normalidade nesses itens também foi identificada na análise dos histogramas. Esses itens se referem a perguntas sobre ideação suicida (D3 "Você tem sentido que a vida não vale a pena?", D4 "Você tem pensado na possibilidade de dar um fim em você mesmo?" e D7 "Você tem achado que a ideia de acabar com a própria vida tem se mantido em sua cabeça?") e, por tal motivo, consideramos que a ausência de normalidade neles é aceitável.

Relativo à fatorabilidade das respostas, examinamos inicialmente os valores das communalities. Encontramos communalities abaixo de 0,30 em quatro itens $(\mathrm{A} 2=0,23$; 
$\mathrm{A} 7=0,21 ; \mathrm{C} 1=0,13 ; \mathrm{C} 2=0,16)$ e acima de $0,70 \mathrm{em} \mathrm{um}$ item $(\mathrm{B} 1=0,73)$. Para esses itens, exploramos a matriz de correlações que indicou boas correlações (entre 0,30 e 0,70$)$ com outros itens, exceto para C1 e C2, itens aos quais optamos por suprimir em todas as análises subsequentes.
Em referência à adequação da amostra, encontramos o KMO de 0,88 e os resultados com o teste de esfericidade de Bartlett $\left(\chi^{2}=3500,74 ; d f=325 ; p<0,001\right)$. A matriz de correlação entre os fatores (Tabela 2) apresentou sete coeficientes maiores ou iguais a 0,30 , corroborando o uso da rotação oblíqua.

Tabela 2

Matriz de Correlações entre os Fatores

\begin{tabular}{|c|c|c|c|c|c|}
\hline Fatores & Ansiedade & $\begin{array}{c}\text { Depressão } \\
\text { Grave }\end{array}$ & $\begin{array}{l}\text { Disfunção } \\
\text { Social }\end{array}$ & Insônia & $\begin{array}{l}\text { Sintomas } \\
\text { Somáticos }\end{array}$ \\
\hline Ansiedade & 1,00 & & & & \\
\hline Depressão grave & $-0,17$ & 1,00 & & & \\
\hline Disfunção social & 0,34 & $-0,38$ & 1,00 & & \\
\hline Insônia & $-0,33$ & 0,39 & $-0,30$ & 1,00 & \\
\hline Sintomas somáticos & 0,46 & $-0,23$ & 0,28 & $-0,41$ & 1,00 \\
\hline
\end{tabular}

O modelo encontrado (Tabela 3) com cinco fatores, explicou 50,96\% da variância. As cargas de cada item foram levantadas (Pattern Matrix) e aquelas $\geq 0,30$ consideradas para a composição de cada fator. Os itens A7 e D5 não obtiveram carga superior a 0,30 em nenhum fator e foram suprimidos na AFC e no cálculo dos alfas de Cronbach. Os fatores foram designados como Ansiedade, Depressão Grave, Disfunção Social, Insônia e Sintomas Somáticos, respectivamente. Na Tabela 3, observamos também que o desequilíbrio de explicação da variância pelos fatores é extenso, de modo que o primeiro fator é muito forte em relação aos demais.

Tabela 3

Cargas Fatoriais (Superiores a 0,30) Extraídas pela Análise Fatorial pela Técnica dos Eixos Principais e Rotação Oblíqua (Pattern Matrix)

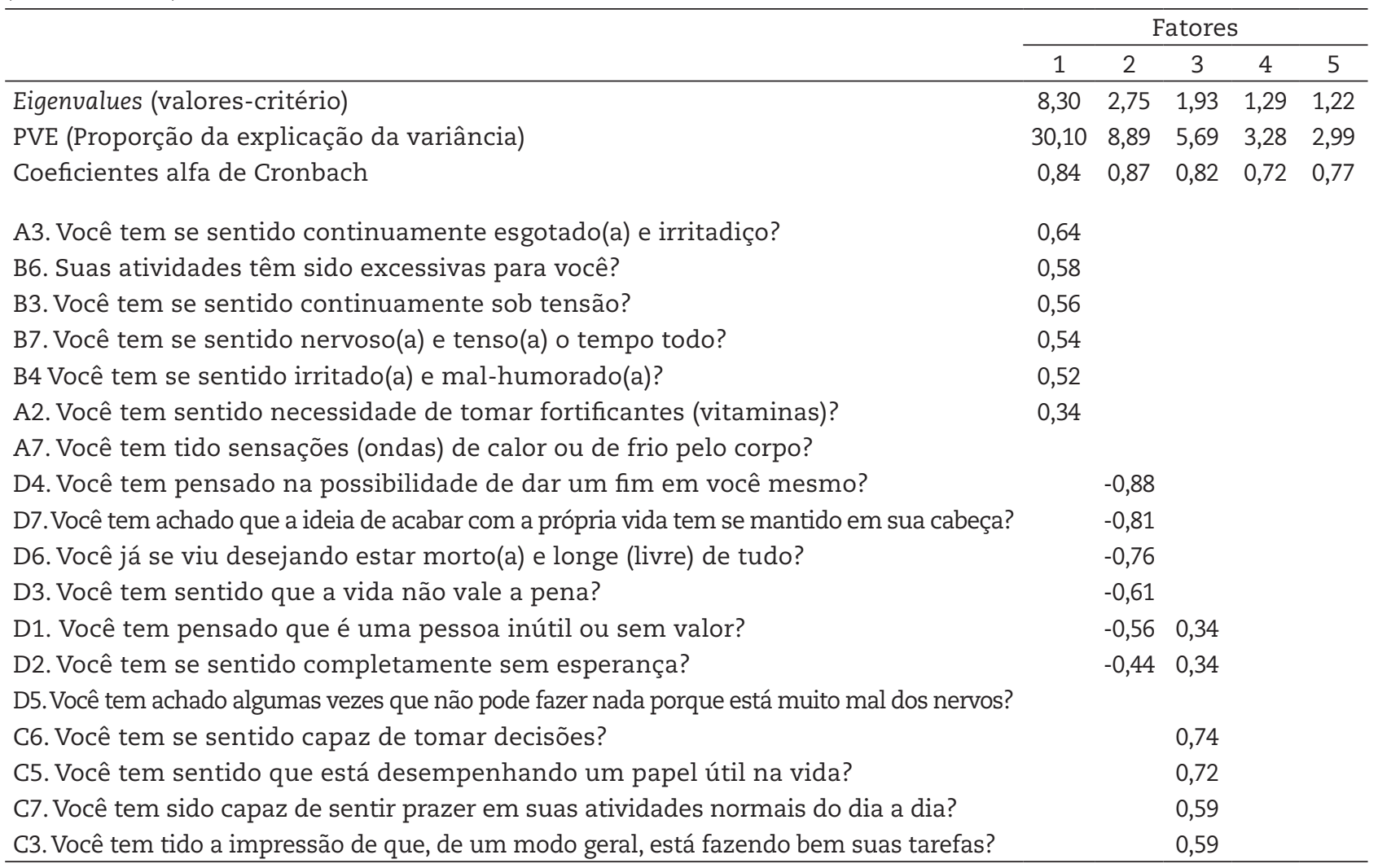


Tabela 3 (continuação)

Cargas Fatoriais (Superiores a 0,30) Extraídas pela Análise Fatorial pela Técnica dos Eixos Principais e Rotação Oblíqua (Pattern Matrix)

\begin{tabular}{|c|c|c|c|c|c|}
\hline & \multicolumn{5}{|c|}{ Fatores } \\
\hline & 1 & 2 & 3 & 4 & 5 \\
\hline C4. Você tem se sentido satisfeito(a) com a forma pela qual tem realizado suas tarefas? & & & 0,58 & & \\
\hline B1. Você tem perdido o sono frequentemente por causa das suas preocupações? & & & & $-0,87$ & \\
\hline B2. Você tem tido dificuldade de continuar dormindo após ter pegado no sono? & & & & $-0,69$ & \\
\hline B5. Você tem ficado assustado(a) ou em pânico sem motivo? & & & & $-0,38$ & \\
\hline A6. Você tem tido sensação de pressão na cabeça ou de que sua cabeça vai estourar? & & & & & 0,83 \\
\hline A5. Você tem sentido dores de cabeça? & & & & & 0,73 \\
\hline A4. Você tem se sentido doente? & & & & & 0,45 \\
\hline A1. Você tem se sentido bem e com boa saúde? & & & & & 0,32 \\
\hline
\end{tabular}

Em relação as AFCs desenvolvidas, na Figura 1 e na Tabela 4, apresentamos a síntese dos resultados acerca da composição fatorial dos modelos e as correlações (Phi, $\Phi$ ) entre os fatores. Observamos na Figura 1 que o modelo de quatro fatores apresentou uma alta correlação $(\Phi=0,84)$ entre os fatores Sintomas Somáticos e Ansiedade e Insônia. O modelo de três fatores apresentou $\Phi=0,62$ entre o fator Estresse Psicológico e Disfunção Social. E o modelo com cinco fatores, três correlações altas $(\Phi=0,62 ; \Phi=0,73 ; \Phi=0,76)$.
Modelo 4 fatores

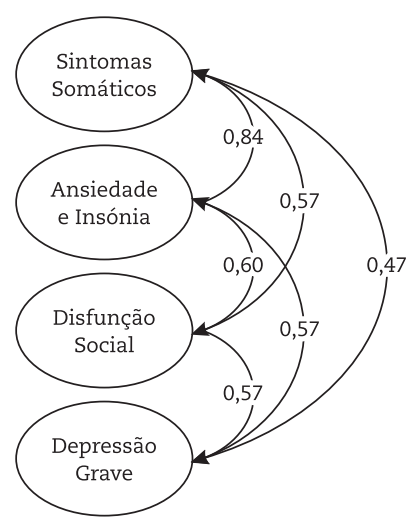

Modelo 3 fatores

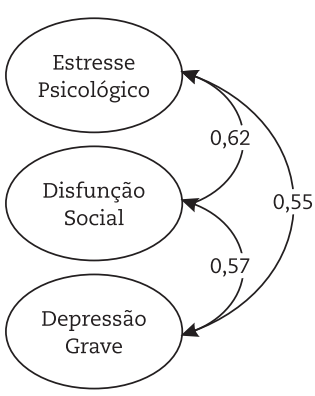

Modelo 5 fatores

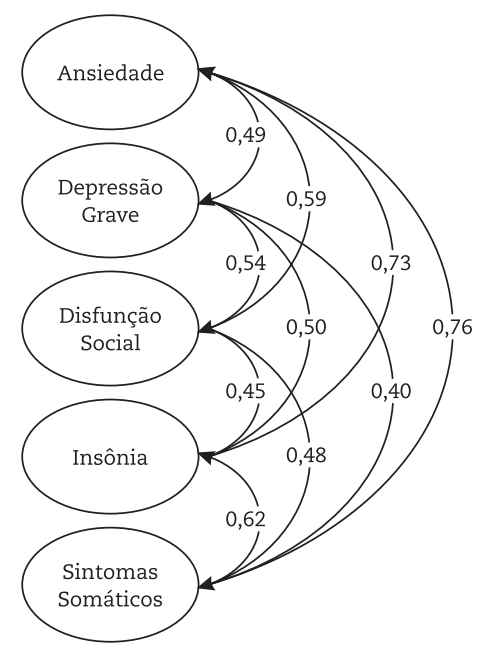

Figura 1. Análise Fatorial Confirmatória dos Modelos com Quatro, Três e Cinco Fatores do QSG-28

Na solução de três fatores, observamos que, se excluímos os três itens com as menores cargas (lambda $<0,40$ ), há melhoria na validade discriminante, pois passa a apresentar: $\Phi=0,59$ entre os fatores de Estresse Psicológico e de Disfunção Social; $\Phi=0,56$ entre Estresse Psicológico e Depressão Grave; e $\Phi=0,57$ entre Disfunção Social e Depressão Grave.

Um bom indicador da qualidade dos modelos foi que nenhum deles apresentou coeficientes de erro maiores que um, variando entre 0,15 e 0,74 no modelo de quatro fatores, entre 0,15 e 0,78 , no modelo de três fatores, e entre 0,14 e 0,80 , no modelo de cinco fatores.

Entretanto as cargas dos itens (lambda) nos fatores variaram entre 0,31 e 0,77 , no modelo de quatro fatores, entre 0,31 e 0,74 , no modelo de três fatores, e entre 0,40 e 0,81 , no modelo de cinco fatores, com 17 itens abaixo de 0,60 , nos modelos de quatro e três fatores, e nove itens abaixo de 0,60 , no modelo de cinco fatores. Por fim, calculamos o alfa de Cronbach para todos os fatores dos modelos considerados, bem como para a escala geral (Tabela 5). 
Tabela 4

Índices de Ajustes da Análise Fatorial Confirmatória do QSG-28 nos Modelos com Quatro, Três e Cinco Fatores

\begin{tabular}{|c|c|c|c|}
\hline $\begin{array}{c}\text { Coeficientes } \\
\text { (respectivos valores de referência*) }\end{array}$ & $\begin{array}{l}\text { Modelo } \\
4 \text { fatores }\end{array}$ & $\begin{array}{l}\text { Modelo } \\
3 \text { fatores }\end{array}$ & $\begin{array}{l}\text { Modelo } \\
5 \text { fatores }\end{array}$ \\
\hline $\begin{array}{l}\text { Qui-quadrado, graus de liberdade } \\
\text { e nível de significância }(p \geq 0,05)\end{array}$ & $\begin{array}{c}\chi^{2}=2061,08 ; d f=344 \\
p<0,001\end{array}$ & $\begin{array}{c}\chi^{2}=2244,00 ; d f=247 \\
p<0,001\end{array}$ & $\begin{array}{c}\chi^{2}=1588,52 ; d f=242 \\
p<0,001\end{array}$ \\
\hline $\begin{array}{l}\text { Qui-quadrado/graus de liberdade } \\
\text { (de } 1 \text { a 3=bom ajuste; até 5=ajuste razoável) }\end{array}$ & $\chi^{2}=/ d f=5,99$ & $\chi^{2}=/ d f=9,08$ & $\chi^{2}=/ d f=6,56$ \\
\hline $\begin{array}{l}\text { Root-Mean-Square Error of Approximation } \\
\text { (ajuste razoável }<0,08 \text {; bom ajuste }<0,05 \text { ) }\end{array}$ & RMSEA $=0,079$ & RMSEA $=0,082$ & RMSEA $=0,084$ \\
\hline Bentler's Comparative Fit Index $(\geq 0,90)$ & $\mathrm{CFI}=0,83$ & $\mathrm{CFI}=0,81$ & CFI $=0,85$ \\
\hline Incremental Fit Index $(\geq 0,90)$ & IFI $=0,83$ & $\mathrm{IFI}=0,81$ & IFI $=0,85$ \\
\hline Relative Fit Index $(\geq 0,90)$ & $R F I=0,78$ & $\mathrm{RFI}=0,76$ & $\mathrm{RFI}=0,80$ \\
\hline Goodness of Fit Index $(\geq 0,90)$ & $\mathrm{GFI}=0,83$ & $\mathrm{GFI}=0,82$ & $\mathrm{GFI}=0,84$ \\
\hline Adjusted GFI $(\geq 0,90)$ & AGFI $=0,80$ & AGFI $=0,79$ & AGFI $=0,80$ \\
\hline $\begin{array}{l}\text { Parsimony GFI } \\
\text { (ajuste razoável } \leq 0,67 \text {; bom ajuste } \leq 0,50 \text { ) }\end{array}$ & PGFI $=0,70$ & PGFI $=0,70$ & PGFI $=0,68$ \\
\hline
\end{tabular}

Nota. *Conforme Malhotra, Lopes, \& Veiga. (2014)

Tabela 5

Alfas de Cronbach ( $\alpha$ ) para os Modelos de Quatro, Três e Cinco Fatores

\begin{tabular}{|c|c|c|c|c|c|}
\hline \multicolumn{2}{|c|}{ Modelo 4 fatores } & \multicolumn{2}{|c|}{ Modelo 3 fatores } & \multicolumn{2}{|c|}{ Modelo 5 fatores } \\
\hline Fator & $\alpha$ & Fator & $\alpha$ & Fator & $\alpha$ \\
\hline Sintomas Somáticos & 0,81 & & & Sintomas Somáticos & 0,77 \\
\hline \multirow{2}{*}{ Ansiedade e Insônia } & \multirow{2}{*}{0,85} & Estresse Psicológico & 0,89 & Ansiedade & 0,84 \\
\hline & & & & Insônia & 0,72 \\
\hline Disfunção Social & 0,80 & Disfunção Social & 0,80 & Disfunção Social & 0,82 \\
\hline Depressão Grave & 0,87 & Depressão Grave & 0,87 & Depressão Grave & 0,87 \\
\hline Geral (28 itens) & 0,92 & Geral (28 itens) & 0,92 & Geral (24 itens) & 0,92 \\
\hline
\end{tabular}

Como a validade discriminante nas AFCs contou com alguns coeficientes Phi desfavoráveis $(\Phi>0,60)$ e a melhor solução na AFE adotou rotação oblíqua, aplicamos também SSA, com a aplicação do Modelo da Distância Euclidiana, que indicou quatro grupos de itens: Depressão Grave $(\alpha=0,87)$; Disfunção Social $(\alpha=0,81)$; Ansiedade $(\alpha=0,85)$ e Sintomas Somáticos e Insônia $(\alpha=0,80)$. Nessa solução (Figura 2), um item previsto como de Sintomas Somáticos (A1 "Você tem se sentido bem e com boa saúde?") agrupou-se com os de Ansiedade, além de que os itens referentes à Insônia se mesclaram com os demais de Sintomas Somáticos. Tal solução pela SSA, além dos coeficientes alfas dentro do desejável (nível de excelência), tem outros indicadores estatísticos satisfatórios: Kruskal's stress=0,14025 e $\mathrm{RSQ}=0,90570$. 


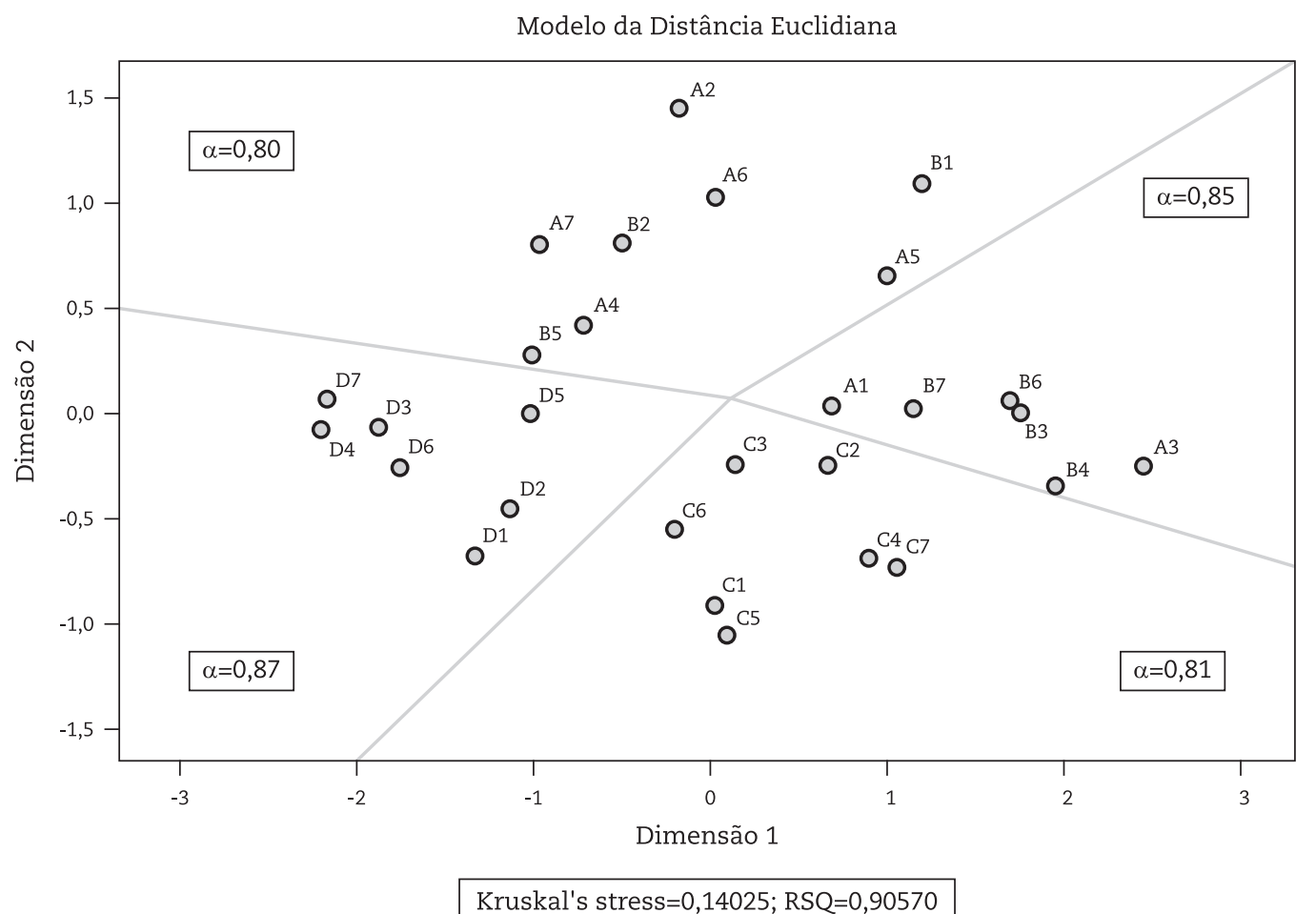

Figura 2. Análise dos itens do QSG-28 pela Aplicação da SSA

\section{Discussão}

A partir dos resultados, constatamos que os coeficientes entre os fatores nos diferentes modelos de AFC indicaram baixa validade discriminante, pois que é tolerável $\Phi \leq 0,60$ (Malhotra, Lopes, \& Veiga, 2014). Observamos que o modelo de quatro fatores apresentou problemas de validade discriminante entre os fatores Sintomas Somáticos e Ansiedade e Insônia. O modelo de três fatores apresentou problemas, em menor medida, entre Estresse Psicológico (junção de Sintomas somáticos e Ansiedade e Insônia) e Disfunção Social, problemas esses que foram superados com a retirada dos itens com lambda $<0,40$. O modelo de cinco fatores seguiu a mesma tendência, com baixa validade discriminante entre Ansiedade e Insônia, entre Ansiedade e Sintomas Somáticos e entre Insônia e Sintomas Somáticos. A falta de validade discriminante do modelo de cinco fatores corrobora a AFE em que se utilizou rotação oblíqua, além de que contou com fatores explicando uma proporção muito baixa da variância. Esses resultados do modelo de cinco fatores demonstraram justamente que a falta de diferenciação está entre os itens que comporiam o fator Estresse Psicológico no modelo de três fatores. E, consequentemente, indicaram que o modelo de três fatores teria melhor validade discriminante que os demais. Esses resultados contrariam a indicação de Werneke et al. (2000) de que uma solução de cinco fatores seria mais indicada para analisar dados brasileiros (mais especificamente cariocas). Entretanto, estão em concordância com Gibbons et al. (2004), Kock et al. (2014) e Werneke et al. (2000), que discorreram sobre a proximidade entre os fatores Sintomas Somáticos e Ansiedade e Insônia, e fortaleceram o uso da solução de três fatores. Todas as soluções encontradas com o uso da AFC não apresentaram bons ajustes, o que provavelmente reflete o fato da normalidade encontrada não atender plenamente ao que é exigido para a aplicação da técnica.

Esses achados em relação à baixa validade discriminante corroboraram a noção de que os fatores são indícios sintomatológicos, e não necessariamente diagnósticos distintos. Vallejo et al. (2014) também mencionaram como desvantagem uma indiferenciação entre problemas físicos e psicológicos pelo instrumento. Segundo Goldberg e Hillier (1979), essa tendência das "subescalas" de intercorrelacionar reflete a presença de um fator geral nos dados não rotacionados. Para o QSG-28, esse fator geral representa $32 \%$ da variância total nos dados de Londres e $37 \%$ para os dois conjuntos de dados de Manchester (Goldberg \& Hillier, 1979). Esses autores, considerando as correlações entre Ansiedade e Insônia e a escala total $(0,90)$, identificaram que a ansiedade é um fenômeno central subjacente às síndromes comuns do transtorno psiquiátrico. Eles justificam que a existência das outras três escalas permite que os pesquisadores com interesses especiais meçam outras dimensões da sintomatologia, com mais informações que as obtidas por um único escore de gravidade. Desse modo, dependendo 
do objetivo para a aplicação do QSG outras versões dele (como a de 12 itens) podem ser uma melhor alternativa, conforme sugerido por Koeter (1992).

Observamos que apenas 11 itens apresentam cargas que atendem aos requisitos de validade convergente $($ lambda $\geq 0,60)$ nos modelos de quatro e três fatores. No modelo de cinco fatores, foram 15 itens que atenderam ao requisito. A eliminação dos itens com cargas inferiores poderia aumentar a validade discriminante e a parcimônia do modelo (Malhotra et al., 2014). Entretanto, consideramos que a exclusão de nove a 17 itens em um instrumento de 28 itens o descaracterizaria e provavelmente impediria a medição de mais dimensões da sintomatologia, que, conforme mencionado anteriormente, seria um ponto forte do QSG-28. Nesse sentido, desde a versão original do QSG-28 são considerados predominantemente os achados clínicos (referentes à validade critério), e não somente os psicométricos, conforme sinalizado por Ribeiro et al. (2015).

De modo geral, os três modelos apresentaram bondade de ajustes na AFC aquém aos descritos na literatura (p. ex., Ardakani et al., 2016; Kock et al., 2014; Munyombwe et al., 2015; Perez et al., 2010). Ainda assim, observamos que o modelo de três fatores após a supressão de três itens (QSG-25) apresentou indicadores ligeiramente melhores que o de quatro e cinco fatores, mas não tão melhores como os encontrados por Perez et al. (2010), por exemplo. Realizamos as AFCs, pois a literatura já citada recomendava a adoção desse caminho, embora que há rejeição da bondade do ajuste pelo qui-quadrado em todos eles (Ver Tabela 1). Além disso, contávamos com um tamanho de amostra que comportaria tais análises. A análise adicional com cinco fatores teve o intuito de investigar mais uma possibilidade, considerando as especificidades brasileiras. No entanto, esse modelo também não obteve ajustes adequados.

A falta de normalidade das respostas a dois itens pode ter contribuído para que os modelos não apresentassem bons ajustes. Entretanto, esses itens dizem respeito a ideação suicida, para os quais a expectativa de normalidade não seja factível nem mesmo na população geral (Botega et al., 2009), nem tomando universitários como um grupo de maior risco (Pereira \& Cardoso, 2017; Veloso et al., 2019). De qualquer forma, recomendamos, em estudos futuros, refletir se a ideação suicida poderia ter outras operacionalizações na forma de itens.

Outro aspecto que não podemos deixar de considerar é a possibilidade de limitações introduzidas pela tradução ao português, apesar do rigor com que aplicamos a técnica de tradução reversa (inglês-português-inglês). Por melhor que seja a tradução do ponto de vista semântico dos itens, ela pode não conseguir mobilizar os mesmos sentidos culturais.

Mesmo considerando as limitações comentadas, os resultados dos alfas de Cronbach podem ser considerados satisfatórios, em especial para os modelos de quatro e três fatores quando aplicado AFC. Os resultados da SSA indicaram que a técnica distingue bem quatro grupos de itens, fortalecendo uma opção de quatro componentes, considerando os indicadores estatísticos, a clareza dos agrupamentos dos itens e os coeficientes alfas obtidos. Devemos ter em conta que a adequação da técnica deve ter haver com as correlações entre fatores referidas na literatura como já comentamos na introdução e nos resultados da AFE.

Desse modo, questionamos a pertinência da utilização indiscriminada de AFC para análise de qualquer instrumento, desconsiderando às vezes as especificidades dos fenômenos psicossociais objeto da mensuração, outras vezes os pressupostos da técnica. O QSG é reconhecido pela sustentação clínica, ou seja, pela sua validade critério (Sá Júnior \& Wang, 2016) no que se refere a sua boa capacidade de realizar triagens, por exemplo, de depressão severa/moderada (Farhood \& Dimassi, 2015) ou para sintomatologia psicológica de pacientes com fibromialgia (Vallejo et al., 2014). A dependência entre os fatores encontrou melhor apresentação na AFE e na SSA do que na AFC. Isso ocorreu porque a AFE prevê o uso de rotação oblíqua, permitindo um ajuste a realidade das respostas quando seus componentes são correlacionados e a SSA é indicada justamente para o caso de lidar-se com variáveis correlacionadas entre si.

Concluímos que: 1. a avaliação da estrutura fatorial do QSG-28 com dados brasileiros contribuem para construir fundamentos científicos para a seleção de instrumentos para usos profissionais e/ou em pesquisas; 2. o modelo de três fatores do QSG-28 se mostrou mais adequado para a avaliação de estudantes universitários, ainda que o modelo de quatro fatores possa ser adotado com base nos resultados da SSA especialmente; 3. o QSG-28 se configura em uma alternativa de instrumento para rastreio de alterações na saúde mental em indivíduos não clínicos, podendo ser utilizado em estudos epidemiológicos e no campo do trabalho; 4) o QSG-28 demanda aperfeiçoamentos para o uso no Brasil que fortaleçam constituir-se em medidas de subescalas de domínios mais específicos da psicopatologia (Gibbons et al., 2004).

\section{Agradecimentos}

Agradecemos ao Prof. Dr. Cornelis Johannes van Stralen pelo apoio.

\section{Financiamento}

A presente pesquisa teve apoio do CNPq por meio de bolsa de Pós-doutorado Júnior - PDJ (Dra. Camila Heleno) e bolsa de produtividade 1A (Dra. Livia Borges).

\section{Contribuições dos autores}

Todos os autores contribuíram substancialmente para a elaboração do delineamento da pesquisa, análise 
e interpretação dos dados, bem como, para a revisão textual e aprovação da versão final deste estudo. Todos os autores assumem responsabilidade pública pelo conteúdo do manuscrito.

\section{Disponibilidade dos dados e materiais}

Todos os dados e sintaxes gerados e analisados durante esta pesquisa serão tratados com total sigilo devido às exigências do Comitê de Ética em Pesquisa em Seres Humanos. Porém, o conjunto de dados e sintaxes que apoiam as conclusões deste artigo estão disponíveis mediante razoável solicitação ao autor principal do estudo.

\section{Conflito de interesses}

Os autores declaram que não há conflitos de interesses.

\section{Referências}

Ardakani, A., Seghatoleslam, T., Habil, H., Jameei, F., Rashid, R., Zahirodin, A.,... \& Arani, A. M. (2016). Construct validity of Symptom Checklist-90-Revised (SCL-90-R) and General Health Questionnaire-28 (GHQ-28) in patients with drug addiction and diabetes, and normal population. Iranian Journal of Public Health, 45(4), 451-459. doi: https://www.ncbi.nlm.nih.gov/pmc/articles/PMC4888172/

Borges, L. O., \& Argolo, J. C. T. (2002). Adaptação e validação de uma escala de bem-estar psicológico para uso em estudos ocupacionais. Avaliação Psicológica, 1(1), 17-27. Recuperado de https://dialnet.unirioja.es/servlet/articulo?codigo $=5115855$

Botega, N. J., Marín-León, L., Oliveira, H. B., Barros, M. B. A., Silva, V. F., \& Dalgalarrondo, P. (2009). Prevalências de ideação, plano e tentativa de suicídio: Um inquérito de base populacional em Campinas, São Paulo, Brasil. Cadernos de Saúde Pública, 25(12), 26322638. doi: 10.1590/S0102-311X2009001200010

Cea D’Ancona, A. (2004). Métodos de encuesta. Teoría y práctica, errores y mejora. Madrid: Editorial Síntesis.

Damásio, B. F. (2012). Uso da análise fatorial exploratória em psicologia. Avaliação Psicológica, 11(2), 213-228. Recuperado de http://pepsic. bvsalud.org/pdf/avp/v11n2/v11n2a07.pdf

Delgado-Gomez, D., Lopez-Castroman, J., Leon-Martinez, V., Baca-Garcia, E., Cabanas-Arrate, M. L., Sanchez-Gonzalez, A., \& Aguado, D. (2013). Psychometrical assessment and item analysis of the General Health Questionnaire in victims of terrorism. Psychological Assessment, 25(1), 279-287. doi: 10.1037/a0030645

Farhood, L. F., \& Dimassi, H. (2015). Validation of an Arabic version of the GHQ-28 against the Beck Depression Inventory for screening for depression in war-exposed civilians. Psychological Reports: Measures E Statitics, 116(2), 470-484. doi: 10.2466/08.PRO.116k23w9

Gibbons, P., Arévalo, H. F., \& Mónico, M. (2004). Assessment of the factor structure and reliability of the 28 item version of the General Health Questionnaire (GHQ-28) in El Salvador. International Journal of Clinical and Health Psychology, 4(2), 389-398.

Goldberg, D. P. (1972). The detection of psychiatric illness by questionnaire. Oxford University Press: London

Goldberg, D. P., \& Hillier, V. F. (1979). A scaled version of the General Health Questionnaire. Psychological Medicine, 9(1), 139-145. doi: 10.1017/S0033291700021644

Goldberg, D. P., Gater, R., Sartorius, N., Üstün, T. B., Piccinelli, M., Gureje, O., \& Rutter, C. (1997). The validity of two versions of the GHQ in the WHO study of mental illness in general health care. Psychological Medicine, 27(1), 191-197. doi: 10.1017/S0033291796004242

Gouveia, V. V., Miranda, F. J., Ramos, A. L. M., Andriola, W. B., \& Pasquali, L. (1996). Questionário de Saúde Geral de Goldberg. Casa do Psicólogo.

Gouveia, V. V., Chaves, S. S. S., Oliveira, I. C. P., Dias, M. R., Gouveia, R. S. V., \& Andrade, P. R. (2003). A utilização do QSG-12 na população geral: Estudo de sua validade de construto. Psicologia: Teoria e Pesquisa, 19(3), 241-248. doi: 10.1590/S0102-37722003000300006

Gray, D. E. (2012). Pesquisa no mundo real (R. C. Costa, Trad.). Porto Alegre: Penso.

Javanmard, G. H., \& Mamaghani, J. (2013). Standardization of GHQ-28 Inventory on the students of Azerbaijan Province of Iran. Procedia - Social and Behavioral Sciences, 84, 47-52. 10.1016/j.sbspro.2013.06.507

Jöreskog, K. G., \& Sörbom, D. (1993). Structural equation modeling with the SIMPLIS Command Language. Lincolnwood: Scientific Software Internacional.

Kock, F. S., Görgens-Ekermans, G., \& Dhladhla, T. J. (2014). A confirmatory factor analysis of the General Health Questionnaire-28 in a Black South African sample. Journal of Health Psychology, 19(10), 1222-1231. doi: 10.1177/1359105313488972

Koeter, M. W. J. (1992). Validity of GHQ and SCL anxiety and depression scales: A comparative study. Journal of Affective Disorders, 24(4), 271-280. doi: 10.1016/0165-0327(92)90112-J

Makowska, Z., Merecz, D., Moscicka, A., \& Kolasa, W. (2002). The validity of General Health Questionnaires, GHQ-12 and GHQ-28, in mental health studies of working people. International Journal of Occupational Medicine and Environmental Health, 15(4), 353-362.

Malakouti, S. K., Fatollahi, P., Mirabzadeh, A., \& Zandi, T. (2006). Reliability, validity and factor structure of the GHQ-28 used among elderly Iranians. International Psychogeriatrics, 19(4), 623-634. doi:10.1017/S1041610206004522

Malhotra, N. K., Lopes, E. L., \& Veiga, R. T. (2014). Modelagem de equações estruturais com o Lisrel: Uma visão inicial. Revista Brasileira de Marketing, 13(2), 28-43. doi: 10.5585/remark.v13i2.2698

Marôco, J. (2014). Análise de equações estruturais: Fundamentos teóricos, software E aplicações. Pêro Pinheiro: Report Number.

Munyombwe, T., West, R. M., \& Hill, K (2015). Testing measurement invariance of the GHQ-28 in stroke patients. Quality of Life Research, 24(8), 1823-1827. doi: 10.1007/s11136-015-0924-8

Pasquali, L., Gouveia, V. V., Andriola, W. B., Miranda, F. J., \& Ramos, A. L. M. (1994). Questionário de Saúde Geral de Goldberg (QSG): Adaptação brasileira. Psicologia: Teoria e Pesquisa, 10(3), 421-438. Recuperado de https://pesquisa.bvsalud.org/brasil/resource/pt/lil156224 ?lang $=$ en

Pereira, A. A. G., \& Cardoso, F. M. S. (2017). Searching for Psychological Predictors of Suicidal Ideation in University Students. Psicologia: Teoria e Pesquisa, 33, e33420. doi: 10.1590/0102.3772e33420 
Pérez, P., Lozano, O. M., \& Rojas, A. J. (2010). Propiedades psicométricas del GHQ-28 em pacientes com dependência a opiáceos. Adicciones, 22(1), 65-72. Recuperado de http://m.adicciones.es/index.php/adicciones/article/view/216

Ribeiro, J. P., Neto, C., Silva, M., Abrantes, C., Coelho, M., Nunes, J, \& Coelho, V. (2015). Ulterior validação do Questionário de Saúde Geral de Goldberg de 28 itens. Psicologia, Saúde E Doenças, 16(3), 278-285. doi: 10.15309/15psd160301

Sá Júnior, A. R., \& Wang, Y. P. (2016). Questionário de Saúde Geral de Goldberg (QSG). Em C. Gorenstein, Y. P. Wang, \& I. Hungerbühler (Orgs.), Instrumentos de avaliação em saúde mental (pp. 77-81). Porto Alegre: Artmed.

Suda, M., Nakayama, K., \& Morimoto, K. (2007). Relationship between behavioral lifestyle and mental health status evaluated using the GHQ-28 and SDS questionnaires in Japanese factory workers. Industrial Health, 45(3), 467-473. doi:10.2486/indhealth.45.467

Üstün, T. B., \& Sartorius, N. (1995). Mental illness in general health care: An international study. Chichester: John Wiley \& Sons.

Tabachnick, B. G., \& Fidell, L. (1989). Using multivariate statistics. New York: Harper-Collins Publishers.

Vallejo, M. A., Rivera, J. Esteve-Vives, J., Rodríguez-Muñoz, M. F., \& ICAF (2014). El cuestionario General de Salud (GHQ-28) en pacientes con fibromialgia: Propiedades psicométricas y adecuación. Clínica y Salud, 25(2), 105-110. doi: 10.1016/j.clysa.2014.06.005

Veloso, L. U. P., Lima, C. L. S., Sales, J. C. S., Monteiro, C. F. S., Gonçalves, A. M. S., \& Silva Júnior, F. J. G. (2019). Ideação suicida em universitários da área da saúde: prevalência e fatores associados. Revista Gaúcha de Enfermagem, 40, e20180144. doi: 10.1590/19831447.2019.20180144

Villano, L. A. B., Nanhay, A. I. G., Moraes, L. R., \& Costa e Silva, J. A. (1995). Results from the Rio de Janeiro Centre. Em T. B. Üstün \& N. Sartorius. Mental illness in general health care: An international study (pp. 227-245). Chichester: John Wiley \& Sons.

Wang, Y. P. (2016). Entrevistas diagnósticas e instrumentos de triagem. Em C. Gorenstein, Y. P. Wang, \& I. Hungerbühler (Orgs.), Instrumentos de avaliação em saúde mental (pp. 59-64). Porto Alegre: Artmed.

Werneke, U., Goldberg, D. P., Yalcin, I., \& Üstün, B. T. (2000). The stability of the factor structure of the General Health Questionnaire. Psychological Medicine, 30(4), 823-829. 10.1017/S0033291799002287

\section{Sobre os autores}

Camila Teixeira Heleno é doutora em Psicologia pela Universidade Federal de Minas Gerais. Atualmente, é professora Adjunta da Universidade Federal dos Vales do Jequitinhonha e Mucuri.

Livia de Oliveira Borges é doutora em Psicologia pela Universidade de Brasília. Atualmente, é professora do Programa de PósGraduação em Psicologia da Universidade Federal de Minas Gerais e bolsista PQ-1A do CNPq.

Esteban Agulló-Tomás é doutor em Ciência Política e Sociologia pela Universidad Complutense de Madrid. Atualmente, é professor da Faculdade de Psicologia da Universidad de Oviedo.

\section{Como citar este artigo}

Heleno et al. (2020). Validade Fatorial do Questionário de Saúde Geral (QSG-28). Avaliação Psicológica, 19(3), 322-332. http://dx.doi. org/10.15689/ap.2020.1903.17586.10 